\title{
Global existence of solutions for a fractional Caputo nonlocal thermistor problem
}

\author{
Moulay Rchid Sidi Ammi ${ }^{1 *}$, Ismail Jamiai ${ }^{1}$ and Delfim F. M. Torres ${ }^{2}$
}

\author{
"Correspondence: sidiammi@ua.pt \\ 'Department of Mathematics, \\ AMNEA Group, Faculty of Sciences \\ and Techniques, Moulay Ismail \\ University, B.P. 509, Errachidia, \\ Morocco \\ Full list of author information is \\ available at the end of the article
}

\begin{abstract}
We begin by proving a local existence result for a fractional Caputo nonlocal thermistor problem. Then additional existence and continuation theorems are obtained, ensuring global existence of solutions.
\end{abstract}

MSC: 26A33; 35A01; 58J20

Keywords: fractional derivatives; fractional PDE; local existence; global existence; fixed point theorem

\section{Introduction}

Fractional calculus is acknowledged as an important research tool that opens up many horizons in the field of dynamical systems [1]. According to Professor Katsuyuki Nishimoto, 'the fractional calculus is the calculus of the XXIst century' [2]. This opinion is strengthened by a huge increase of interest in this research tool, expressed by an increase in the number of theoretical developments and basic theory on this subject; see, e.g., [3-7]. Recently, it has also been proved that fractional differential equations are significant and essential tools when applied in the study of nonlocal or time-dependent processes and in the modeling of many applications, including chaotic dynamics, material sciences, mechanic of fractal and complex media, quantum mechanics, physical kinetics, chemistry, biology, economics and control theory [8]. For instance, a fractional generalization of the Newtonian equation to describe the dynamics of complex phenomena, in both science and engineering, has been proposed in [9]; a fractional Langevin equation, with applications in polymer layers, has been investigated in [10]. One can say that real-world problems require the definitions of fractional derivatives for initial and boundary value problems $[11,12]$. Fractional mathematical models describing natural phenomena, like shallow water waves and ion acoustic waves in plasma and vibration of large membranes, as well as personal and interpersonal realities, like smoking, romantic relationships and marriages, can be found in $[13,14]$ and $[15,16]$, respectively. Details of the geometric and physical interpretation of fractional differentiation can be found in [6].

Thermistor is a thermo-electric device constructed from a ceramic material whose electrical conductivity depends strongly on the temperature. This makes thermistor problems highly nonlinear [17]. They can be used as a switching device in many electronic circuits.

(c) The Author(s) 2017. This article is distributed under the terms of the Creative Commons Attribution 4.0 International License (http://creativecommons.org/licenses/by/4.0/), which permits unrestricted use, distribution, and reproduction in any medium, provided you give appropriate credit to the original author(s) and the source, provide a link to the Creative Commons license, and indicate if changes were made. 
A broad application spectrum of thermistor problems in heating processes and current flow can be found in several areas of electronics and its related industries [18]. Generally, there are two kinds of thermistors: the first have an electrical conductivity that decreases with the increasing of temperature; the second have an electrical conductivity that increases with the increasing of temperature $[19,20]$. Here we consider a prototype of electrical conductivity that depends strongly in both time and temperature. Our goal consists in proving global existence of solutions for a fractional Caputo nonlocal thermistor problem. The results are obtained via Schauder's fixed point theorem. Precisely, we consider the following fractional order initial value problem:

$$
\begin{aligned}
& { }_{C} D_{0, t}^{2 \alpha} u(t)=\frac{\lambda f(t, u(t))}{\left(\int_{0}^{t} f(x, u(x)) d x\right)^{2}}, \quad t \in(0, \infty), \\
& \left.u(t)\right|_{t=0}=u_{0},
\end{aligned}
$$

where ${ }_{C} D_{0, t}^{2 \alpha}$ is the fractional Caputo derivative operator of order $2 \alpha$ with $0<\alpha<\frac{1}{2}$ a real parameter. The function $u$ denotes the temperature and $\lambda$ is a positive real. We shall assume the following hypotheses:

$\left(H_{1}\right) f: \mathbb{R}^{+} \times \mathbb{R}^{+} \rightarrow \mathbb{R}^{+}$is a Lipschitz continuous function with Lipschitz constant $L_{f}$ with respect to the second variable such that $c_{1} \leq f(s, u) \leq c_{2}$ with $c_{1}$ and $c_{2}$ two positive constants;

$\left(H_{2}\right)$ there exists a positive constant $M$ such that $f(s, u) \leq M s^{2}$;

$\left(H_{3}\right)|f(s, u)-f(s, v)| \leq s^{2}|u-v|$ or, in a more general manner, there exists a constant $\omega \geq 2$ such that $|f(s, u)-f(s, v)| \leq s^{\omega}|u-v|$.

In the literature, questions involving the existence and uniqueness of solution for fractional differential equations (FDEs) have been intensely studied by many mathematicians $[4,5,21,22]$. However, much of published papers have been concerned with existenceuniqueness of solutions for FDEs on a finite interval. Since continuation theorems for FDEs are not well developed, results as regards global existence-uniqueness of the solution of FDEs on the half axis $[0,+\infty)$, by using directly the results from local existence, have only recently flourished $[23,24]$.

In contrast with our previous work [25-27] on fractional nonlocal thermistor problems, which was focused on local existence and numerical methods, here we are concerned with continuation theorems and global existence for the steady state fractional Caputo nonlocal thermistor problem. The paper is organized as follows. In Section 2, we collect some background material and necessary results from fractional calculus. Then we are concerned in Section 3 with local existence on a finite interval for (1) (Theorem 3.2). Section 4 is devoted to the (non-)continuation (Theorem 4.1) associated with problem (1), which allows one to generalize the main result of Section 3. Our proofs rely on Schauder's fixed point theorem and some extensions of the continuation theorems for ordinary differential equations (ODEs) to the fractional order case. One of the main difficulties lies in handling the nonlocal term $\frac{\lambda f(t, u(t))}{\left(\int_{0}^{t} f(x, u(x)) d x\right)^{2}}$, representing a heat source and that depends continuously on time; another one in the fact that electrical conductivity depends on both time and temperature. Based on the results of Section 4, in Section 5 we prove existence of a global solution for (1): see Theorems 5.2 and 5.3. We end with Section 6 presenting conclusions. 


\section{Preliminaries and basic results}

In this section, we collect from the literature $[4,5,22,28-30]$ some background material and basic results that will be used in the remainder of the paper.

Let $C[a, b]$ be the Banach space of all real valued continuous functions on $[a, b]$ endowed with the norm $\|x\|_{[a, b]}=\max _{t \in[a, b]}|x(t)|$. According to the Riemann-Liouville approach to fractional calculus, we introduce the fractional integral of order $\alpha, \alpha>0$, as follows.

Definition 2.1 The Riemann-Liouville integral of a function $g$ with order $\alpha>0$ is defined by

$$
{ }_{R L} D_{0, t}^{-\alpha} g(t)=\frac{1}{\Gamma(\alpha)} \int_{0}^{t}(t-s)^{\alpha-1} g(s) d s, \quad t>0,
$$

where $\Gamma$ is the Euler gamma function given by

$$
\Gamma(\alpha)=\int_{0}^{\infty} t^{\alpha-1} e^{-t} d t
$$

$\alpha>0$.

The natural next step, after the notion of fractional integral has been introduced, is to define the fractional derivative of order $\alpha, \alpha>0$.

Definition 2.2 The Riemann-Liouville derivative of the function $g$ with order $\alpha>0$ is defined by

$$
{ }_{R L} D_{0, t}^{\alpha} g(t)=\frac{1}{\Gamma(n-\alpha)} \frac{d^{n}}{d t^{n}} \int_{0}^{t}(t-s)^{n-\alpha-1} g(s) d s, \quad t>0,
$$

where $n-1<\alpha<n \in \mathbb{Z}^{+}$.

Note the remarkable fact that, in the Riemann-Liouville sense, the fractional derivative of the constant function is not zero. We now give an alternative and more restrictive definition of fractional derivative, first introduced by Caputo in the end of the 1960s [31, $32]$ and then adopted by Caputo and Mainardi in [33, 34]. In Caputo sense, the fractional derivative of a constant is zero.

Definition 2.3 The Caputo derivative of the function $g(t)$ with order $\alpha>0$ is defined by

$$
{ }_{C} D_{0, t}^{\alpha} g(t)=\frac{1}{\Gamma(n-\alpha)} \int_{0}^{t}(t-s)^{\alpha-1} g^{(n)}(s) d s, \quad t>0,
$$

where $n-1<\alpha<n \in \mathbb{Z}^{+}$.

For proving our main results, we make use of the following auxiliary lemmas.

Lemma 2.1 (See [24]) Let $M$ be a subset of $C([0, T])$. Then $M$ is precompact if and only if the following conditions hold: 
1. $\{u(t): u \in M\}$ is uniformly bounded,

2. $\{u(t): u \in M\}$ is equicontinuous on $[0, T]$.

Lemma 2.2 (Schauder fixed point theorem [24]) Let U be a closed bounded convex subset of a Banach space $X$. If $T: U \rightarrow U$ is completely continuous, then $T$ has a fixed point in $U$.

Finally we recall a generalization of Gronwall's lemma, which is essential for the proof of our Theorem 5.3.

Lemma 2.3 (Generalized Gronwall inequality $[35,36]$ ) Let $v:[0, b] \rightarrow[0,+\infty)$ be a real function and $w(\cdot)$ be a nonnegative, locally integrable function on $[0, b]$. Suppose that there exist $a>0$ and $0<\alpha<1$ such that

$$
v(t) \leq w(t)+a \int_{0}^{t} \frac{v(s)}{(t-s)^{\alpha}} d s .
$$

Then there exists a constant $k=k(\alpha)$ such that

$$
v(t) \leq w(t)+k a \int_{0}^{t} \frac{w(s)}{(t-s)^{\alpha}} d s
$$

for $t \in[0, b]$.

\section{Local existence theorem}

In this section, a local existence theorem of solutions for (1) is obtained by applying Schauder's fixed point theorem. In order to transform (1) into a fixed point problem, we give in the following lemma an equivalent integral form of (1).

Lemma 3.1 Suppose that $\left(H_{1}\right)-\left(H_{3}\right)$ holds. Then the initial value problem (1) is equivalent to

$$
u(t)=u_{0}+\frac{\lambda}{\Gamma(2 \alpha)} \int_{0}^{t}(t-s)^{2 \alpha-1} \frac{f(s, u(s))}{\left(\int_{0}^{t} f(x, u) d x\right)^{2}} d s
$$

Proof It is a simple exercise to see that $u$ is a solution of the integral equation (2) if and only if it is also a solution of the IVP (1).

Theorem 3.2 Suppose that conditions $\left(H_{1}\right)-\left(H_{3}\right)$ are verified. Then (1) has at least one solution $u \in C[0, h]$ for some $T \geq h>0$.

Proof Let

$$
E=\left\{u \in C[0, T]:\left\|u-u_{0}\right\|_{C[0, T]}=\sup _{0 \leq t \leq T}\left|u-u_{0}\right| \leq b\right\},
$$

where $b$ is a positive constant. Further, put

$$
D_{h}=\left\{u: u \in C[0, h],\left\|u-u_{0}\right\|_{C[0, h]} \leq b\right\},
$$


where

$$
h=\min \left\{\left(b\left(\frac{\lambda M}{\Gamma(2 \alpha+1) c_{1}^{2}}\right)^{-1}\right)^{\frac{1}{2 \alpha}}, T\right\}
$$

and $0<\alpha<\frac{1}{2}$. It is clear that $h \leq T$. Note also that $D_{h}$ is a nonempty, bounded, closed, and convex subset of $C[0, h]$. In order to apply Schauder's fixed point theorem, we define the following operator $A$ :

$$
(A u)(t)=u_{0}+\frac{\lambda}{\Gamma(2 \alpha)} \int_{0}^{t}(t-s)^{2 \alpha-1} \frac{f(s, u(s))}{\left(\int_{0}^{t} f(x, u) d x\right)^{2}} d s, \quad t \in[0, h] .
$$

It is clear that all solutions of (1) are fixed points of (3). Then, by assumptions $\left(H_{1}\right)$ and $\left(H_{2}\right)$, we have for any $u \in C[0, h]$

$$
\begin{aligned}
\left|(A u)(t)-u_{0}\right| & \leq \frac{\lambda}{\Gamma(2 \alpha)} \frac{1}{\left(c_{1} t\right)^{2}} \int_{0}^{t}(t-s)^{2 \alpha-1} f(s, u(s)) d s \\
& \leq \frac{\lambda}{\Gamma(2 \alpha)} \frac{M}{c_{1}^{2}} \int_{0}^{t}(t-s)^{2 \alpha-1} d s \\
& \leq \frac{\lambda M}{2 \alpha \Gamma(2 \alpha)} \frac{1}{c_{1}^{2}} h^{2 \alpha} \\
& =\frac{\lambda M}{\Gamma(2 \alpha+1)} \frac{1}{c_{1}^{2}} h^{2 \alpha} \\
& \leq b
\end{aligned}
$$

It yields $A D_{h} \subset D_{h}$. Our next step, in order to prove Theorem 3.2, is to show that the following lemma holds.

Lemma 3.3 The operator $A$ is continuous.

Proof Let $u_{n}, u \in D_{h}$ be such that $\left\|u_{n}-u\right\|_{C[0, h]} \rightarrow 0$ as $n \rightarrow+\infty$. One has

$$
\begin{aligned}
&\left|A u_{n}(t)-A u(t)\right| \\
& \leq \frac{\lambda}{\Gamma(2 \alpha)} \int_{0}^{t}(t-s)^{2 \alpha-1}\left|\frac{f\left(s, u_{n}(s)\right)}{\left(\int_{0}^{t} f\left(x, u_{n}\right) d x\right)^{2}}-\frac{f(s, u(s))}{\left(\int_{0}^{t} f(x, u) d x\right)^{2}}\right| d s \\
& \leq \frac{\lambda}{\Gamma(2 \alpha)} \int_{0}^{t}(t-s)^{2 \alpha-1} \mid \frac{1}{\left(\int_{0}^{t} f\left(x, u_{n}\right) d x\right)^{2}}\left(f\left(s, u_{n}(s)\right)-f(s, u(s))\right) \\
&+f(s, u(s))\left(\frac{1}{\left(\int_{0}^{t} f\left(x, u_{n}\right) d x\right)^{2}}-\frac{1}{\left(\int_{0}^{t} f(x, u) d x\right)^{2}}\right) \mid d s \\
& \leq \frac{\lambda}{\Gamma(2 \alpha)} \int_{0}^{t}(t-s)^{2 \alpha-1} \frac{1}{\left(\int_{0}^{t} f\left(x, u_{n}\right) d x\right)^{2}}\left|f\left(s, u_{n}(s)\right)-f(s, u(s))\right| d s \\
& \quad+\frac{\lambda}{\Gamma(2 \alpha)} \int_{0}^{t}(t-s)^{2 \alpha-1}|f(s, u(s))|\left|\frac{1}{\left(\int_{0}^{t} f\left(x, u_{n}\right) d x\right)^{2}}-\frac{1}{\left(\int_{0}^{t} f(x, u) d x\right)^{2}}\right| \\
& \leq
\end{aligned}
$$


We now focus on both right hand terms separately. By hypotheses $\left(H_{2}\right)$ and $\left(H_{3}\right)$, we have

$$
\begin{aligned}
I_{1} & \leq \frac{\lambda}{\left(c_{1} t\right)^{2} \Gamma(2 \alpha)} \int_{0}^{t}(t-s)^{2 \alpha-1}\left|f\left(s, u_{n}(s)\right)-f(s, u(s))\right| d s \\
& \leq \frac{\lambda L_{f}}{c_{1}^{2} \Gamma(2 \alpha)} \int_{0}^{t}(t-s)^{2 \alpha-1}\left|u_{n}(s)-u(s)\right| d s \\
& \leq \frac{\lambda L_{f}}{c_{1}^{2} \Gamma(2 \alpha)}\left\|u_{n}-u\right\|_{C[0, h]} \int_{0}^{t}(t-s)^{2 \alpha-1} d s \\
& \leq \frac{\lambda L_{f}}{c_{1}^{2} \Gamma(2 \alpha)}\left\|u_{n}-u\right\|_{C[0, h]} \int_{0}^{t}(t-s)^{2 \alpha-1} d s .
\end{aligned}
$$

Then

$$
I_{1} \leq \frac{\lambda h^{2 \alpha} L_{f}}{c_{1}^{2} \Gamma(2 \alpha+1)}\left\|u_{n}-u\right\|_{C[0, h]} .
$$

Concerning the second term, we have

$$
\begin{aligned}
I_{2} \leq & \frac{\lambda}{\Gamma(2 \alpha)} \int_{0}^{t} \frac{(t-s)^{2 \alpha-1}|f(s, u(s))|}{\left(\int_{0}^{t} f\left(x, u_{n}\right) d x\right)^{2}\left(\int_{0}^{t} f(x, u) d x\right)^{2}} \\
& \times\left|\left(\int_{0}^{t} f\left(x, u_{n}\right) d x\right)^{2}-\left(\int_{0}^{t} f(x, u) d x\right)^{2}\right| d s \\
\leq & \frac{\lambda c_{2}}{\left(c_{1} t\right)^{4} \Gamma(2 \alpha)} \int_{0}^{t}(t-s)^{2 \alpha-1}\left|\left(\int_{0}^{t} f\left(x, u_{n}\right) d x\right)^{2}-\left(\int_{0}^{t} f(x, u) d x\right)^{2}\right| d s \\
\leq & \frac{\lambda c_{2}}{\left(c_{1} t\right)^{4} \Gamma(2 \alpha)} \int_{0}^{t}(t-s)^{2 \alpha-1} \\
& \times\left|\left(\int_{0}^{t}\left(f\left(x, u_{n}\right)-f(x, u)\right) d x\right)\left(\int_{0}^{t}\left(f\left(x, u_{n}\right)+f(x, u)\right) d x\right)\right| d s \\
\leq & \frac{2 \lambda c_{2}^{2} t}{\left(c_{1} t\right)^{4} \Gamma(2 \alpha)} \int_{0}^{t}(t-s)^{2 \alpha-1}\left(\int_{0}^{t}\left|f\left(x, u_{n}\right)-f(x, u)\right| d x\right) d s \\
\leq & \frac{2 \lambda c_{2}^{2} t^{3} L_{f}}{\left(c_{1} t\right)^{4} \Gamma(2 \alpha)} \int_{0}^{t}(t-s)^{2 \alpha-1}\left(\int_{0}^{t}\left|u_{n}(x)-u(x)\right| d x\right) d s \\
\leq & \frac{2 \lambda c_{2}^{2} t^{4} L_{f}}{\left(c_{1} t\right)^{4} \Gamma(2 \alpha)}\left\|u_{n}-u\right\|_{C[0, h]} \int_{0}^{t}(t-s)^{2 \alpha-1} d s \\
\leq & \frac{2 \lambda c_{2}^{2} L_{f}}{c_{1}^{4} \Gamma(2 \alpha)}\left\|u_{n}-u\right\|_{C[0, h]} \int_{0}^{t}(t-s)^{2 \alpha-1} d s \\
\leq & \frac{2 \lambda c_{2}^{2} h^{2 \alpha} L_{f}}{c_{1}^{4} \Gamma(2 \alpha+1)}\left\|u_{n}-u\right\|_{C[0, h]} .
\end{aligned}
$$

It follows that

$$
I_{2} \leq \frac{2 \lambda c_{2}^{2} h^{2 \alpha} L_{f}}{c_{1}^{4} \Gamma(2 \alpha+1)}\left\|u_{n}-u\right\|_{C[0, h]} .
$$


Collecting inequalities (5) and (6) together, and inserting into (4), we have

$$
\begin{aligned}
\left|A u_{n}(t)-A u(t)\right| & \leq I_{1}+I_{2} \\
& \leq\left(\frac{\lambda h^{2 \alpha} L_{f}}{c_{1}^{2} \Gamma(2 \alpha+1)}+\frac{2 \lambda c_{2}^{2} h^{2 \alpha} L_{f}}{c_{1}^{4} \Gamma(2 \alpha+1)}\right)\left\|u_{n}-u\right\|_{C[0, h]} .
\end{aligned}
$$

Therefore,

$$
\left\|A u_{n}-A u\right\|_{C[0, h]} \leq\left(\frac{\lambda h^{2 \alpha} L_{f}}{c_{1}^{2} \Gamma(2 \alpha+1)}+\frac{2 \lambda c_{2}^{2} h^{2 \alpha} L_{f}}{c_{1}^{4} \Gamma(2 \alpha+1)}\right)\left\|u_{n}-u\right\|_{C[0, h]} .
$$

Consequently, $\left\|A u_{n}-A u\right\|_{C[0, h]} \rightarrow 0$ as $n \rightarrow+\infty$, which proves that $A$ is continuous. This ends the proof of Lemma 3.3.

To finish the proof of Theorem 3.2, it remains to show the following.

Lemma 3.4 The operator $A D_{h}$ is continuous.

Proof Let $u \in D_{h}$ and $0 \leq t_{1} \leq t_{2} \leq h$. Then

$$
\begin{aligned}
&\left|(A u)\left(t_{2}\right)-(A u)\left(t_{1}\right)\right| \\
& \leq \frac{\lambda}{\Gamma(2 \alpha)}\left|\int_{0}^{t_{2}}\left(t_{2}-s\right)^{2 \alpha-1} \frac{f(s, u(s))}{\left(\int_{0}^{t_{2}} f(x, u) d x\right)^{2}} d s-\int_{0}^{t_{1}}\left(t_{1}-s\right)^{2 \alpha-1} \frac{f(s, u(s))}{\left(\int_{0}^{t_{1}} f(x, u) d x\right)^{2}} d s\right| \\
& \leq \frac{\lambda}{\Gamma(2 \alpha)} \mid \int_{0}^{t_{1}}\left(\left(t_{1}-s\right)^{2 \alpha-1}-\left(t_{2}-s\right)^{2 \alpha-1}\right) \frac{f(s, u(s))}{\left(\int_{0}^{t_{1}} f(x, u) d x\right)^{2}} d s \\
& \quad+\int_{0}^{t_{1}}\left(t_{2}-s\right)^{2 \alpha-1} \frac{f(s, u(s))}{\left(\int_{0}^{t_{1}} f(x, u) d x\right)^{2}} d s-\int_{0}^{t_{2}}\left(t_{2}-s\right)^{2 \alpha-1} \frac{f(s, u(s))}{\left(\int_{0}^{t_{2}} f(x, u) d x\right)^{2}} d s \mid \\
& \leq \frac{\lambda}{\Gamma(2 \alpha)} \mid \int_{0}^{t_{1}}\left(\left(t_{1}-s\right)^{2 \alpha-1}-\left(t_{2}-s\right)^{2 \alpha-1}\right) \frac{f(s, u(s))}{\left(\int_{0}^{t_{1}} f(x, u) d x\right)^{2}} d s \\
& \quad+\int_{0}^{t_{1}}\left(t_{2}-s\right)^{2 \alpha-1} f(s, u(s))\left(\frac{1}{\left(\int_{0}^{t_{1}} f(x, u) d x\right)^{2}}-\frac{1}{\left(\int_{0}^{t_{2}} f(x, u) d x\right)^{2}}\right) d s \\
& \quad-\int_{t_{1}}^{t_{2}}\left(t_{2}-s\right)^{2 \alpha-1} \frac{f(s, u(s))}{\left(\int_{0}^{t_{2}} f(x, u) d x\right)^{2}} d s \mid \\
& \leq I_{3}+I_{4}+I_{5},
\end{aligned}
$$

where we have, by direct calculation,

$$
\begin{aligned}
I_{3} & \leq \frac{\lambda c_{2}}{\left(c_{1} t_{1}\right)^{2} \Gamma(2 \alpha)}\left|\int_{0}^{t_{1}}\left(\left(t_{1}-s\right)^{2 \alpha-1}-\left(t_{2}-s\right)^{2 \alpha-1}\right) d s\right| \\
& \leq \frac{\lambda c_{2}}{\left(c_{1} t_{1}\right)^{2} \Gamma(2 \alpha+1)}\left|t_{1}^{2 \alpha}-t_{2}^{2 \alpha}+\left(t_{2}-t_{1}\right)^{2 \alpha}\right|, \\
I_{4} & \leq \frac{\lambda}{\Gamma(2 \alpha)} \frac{c_{2} t_{2}^{2 \alpha-1}}{\left(c_{1} t_{1}\right)^{2}\left(c_{1} t_{2}\right)^{2}} \int_{0}^{t_{1}}\left|\left(\int_{0}^{t_{2}} f(x, u) d x\right)^{2}-\left(\int_{0}^{t_{1}} f(x, u) d x\right)^{2}\right| d s \\
& \leq \frac{\lambda}{\Gamma(2 \alpha)} \frac{c_{2} t_{2}^{2 \alpha-1}}{\left(c_{1} t_{1}\right)^{2}\left(c_{1} t_{2}\right)^{2}}
\end{aligned}
$$




$$
\begin{aligned}
& \times \int_{0}^{t_{1}}\left|\left(\int_{0}^{t_{2}} f(x, u) d x+\int_{0}^{t_{1}} f(x, u) d x\right)\left(\int_{0}^{t_{2}} f(x, u) d x-\int_{0}^{t_{1}} f(x, u) d x\right)\right| d s \\
\leq & \frac{\lambda}{\Gamma(2 \alpha)} \frac{c_{2}^{2}\left(t_{1}+t_{2}\right) t_{2}^{2 \alpha-1}}{\left(c_{1} t_{1}\right)^{2}\left(c_{1} t_{2}\right)^{2}}\left|\int_{t_{1}}^{t_{2}} f(s, u(s)) d s\right| \\
\leq & \frac{\lambda}{\Gamma(2 \alpha)} \frac{c_{2}^{3}\left(t_{1}+t_{2}\right) t_{2}^{2 \alpha-1}}{\left(c_{1} t_{1}\right)^{2}\left(c_{1} t_{2}\right)^{2}}\left|t_{2}-t_{1}\right|,
\end{aligned}
$$

and

$$
I_{5} \leq \frac{\lambda c_{2}}{\left(c_{1} t_{2}\right)^{2} \Gamma(2 \alpha)} \int_{t_{1}}^{t_{2}}\left(t_{2}-s\right)^{2 \alpha-1} d s \leq \frac{\lambda c_{2}}{\left(c_{1} t_{2}\right)^{2} \Gamma(2 \alpha+1)}\left(t_{2}-t_{1}\right)^{2 \alpha} .
$$

The right hand side of inequalities (9) and (10) do not depend on $u$ and converge to zero as $t_{2} \rightarrow t_{1}$. Then $\left\{(A u)(t): u \in D_{h}\right\}$ is equicontinuous and Lemma 3.4 is proved.

Taking into account that $A D_{h} \subset D_{h}$, we infer that $A D_{h}$ is precompact. This implies that $A$ is completely continuous. As a consequence of Schauder's fixed point theorem and Lemma 3.1, we conclude that problem (1) has a local solution. This ends the proof of Theorem 3.2.

\section{Continuation results}

Our main contribution of this section is to prove a continuation theorem for the fractional Caputo nonlocal thermistor problem (1). First, we present the definition of noncontinuable solution.

Definition 4.1 (See [37]) Let $u(t)$ on $(0, \beta)$ and $\tilde{u}(t)$ on $(0, \tilde{\beta})$ be both solutions of (1). If $\beta<\tilde{\beta}$ and $u(t)=\tilde{u}(t)$ for $t \in(0, \beta)$, then we say that $\tilde{u}(t)$ can be continued to $(0, \tilde{\beta})$. A solution $u(t)$ is noncontinuable if it has no continuation. The existing interval of the noncontinuable solution $u(t)$ is called the maximum existing interval of $u(t)$.

Theorem 4.1 Assume that conditions $\left(H_{1}\right)-\left(H_{3}\right)$ are satisfied. Then $u=u(t), t \in(0, \beta)$, is noncontinuable if and if only for some $\eta \in\left(0, \frac{\beta}{2}\right)$ and any bounded closed subset $S \subset$ $[\eta,+\infty) \times \mathbb{R}$ there exists a $t^{*} \in[\eta, \beta)$ such that $\left(t^{*}, u\left(t^{*}\right)\right) \notin S$.

Proof Suppose that there exists a compact subset $S \subset[\eta,+\infty) \times \mathbb{R}$ such that

$$
\{(t, u(t)): t \in[\eta, \beta)\} \subset S .
$$

The compactness of $S$ implies $\beta<+\infty$. The remainder of the proof is given in two lemmas.

Lemma 4.2 The limit $\lim _{t \rightarrow \beta^{-}} u(t)$ exists.

Proof Let $t_{1}, t_{2} \in[2 \eta, \beta)$ such that $t_{1}<t_{2}$. From (8), we have

$$
I_{3}=I_{3,1}+I_{3,2},
$$

where

$$
I_{3,1}=\frac{\lambda}{\Gamma(2 \alpha)} \int_{0}^{\eta}\left(\left(t_{1}-s\right)^{2 \alpha-1}-\left(t_{2}-s\right)^{2 \alpha-1}\right) \frac{f(s, u(s))}{\left(\int_{0}^{t_{1}} f(x, u) d x\right)^{2}} d s
$$


and

$$
I_{3,2}=\frac{\lambda}{\Gamma(2 \alpha)} \int_{\eta}^{t_{1}}\left(\left(t_{1}-s\right)^{2 \alpha-1}-\left(t_{2}-s\right)^{2 \alpha-1}\right) \frac{f(s, u(s))}{\left(\int_{0}^{t_{1}} f(x, u) d x\right)^{2}} d s .
$$

Under assumptions $\left(H_{1}\right)-\left(H_{3}\right)$, there exists a positive constant $M_{3,1}$ such that

$$
\begin{aligned}
I_{3,1} & \leq M_{3,1} \int_{0}^{\eta}\left(\left(t_{1}-s\right)^{2 \alpha-1}-\left(t_{2}-s\right)^{2 \alpha-1}\right) \\
& \leq M_{3,1}\left|\left(t_{2}-\eta\right)^{2 \alpha}-\left(t_{1}-\eta\right)^{2 \alpha}+t_{1}^{2 \alpha}-t_{2}^{2 \alpha}\right| .
\end{aligned}
$$

Moreover, there exists a positive constant $M_{3,2}$ such that

$$
\begin{aligned}
I_{3,2} & \leq M_{3,2} \int_{\eta}^{t_{1}}\left(\left(t_{1}-s\right)^{2 \alpha-1}-\left(t_{2}-s\right)^{2 \alpha-1}\right) \\
& \leq M_{3,2}\left|\left(t_{2}-t_{1}\right)^{2 \alpha}+\left(t_{1}-\eta\right)^{2 \alpha}-\left(t_{1}-\eta\right)^{2 \alpha}\right|
\end{aligned}
$$

and we also have $I_{4}=I_{4,1}+I_{4,2}$, where

$$
I_{4,1}=\int_{0}^{\eta}\left(t_{2}-s\right)^{2 \alpha-1} f(s, u(s))\left(\frac{1}{\left(\int_{0}^{t_{1}} f(x, u) d x\right)^{2}}-\frac{1}{\left(\int_{0}^{t_{2}} f(x, u) d x\right)^{2}}\right) d s
$$

and

$$
I_{4,2}=\int_{\eta}^{t_{1}}\left(t_{2}-s\right)^{2 \alpha-1} f(s, u(s))\left(\frac{1}{\left(\int_{0}^{t_{1}} f(x, u) d x\right)^{2}}-\frac{1}{\left(\int_{0}^{t_{2}} f(x, u) d x\right)^{2}}\right) d s .
$$

In the same manner as in the proof of Lemma 3.4, there exists positive constants $M_{4,1}$ and $M_{4,2}$ such that

$$
\begin{aligned}
& I_{4,1} \leq M_{4,1}\left|t_{2}-t_{1}\right|, \\
& I_{4,2} \leq M_{4,2}\left|t_{2}-t_{1}\right| .
\end{aligned}
$$

We have already proved in (10), for some positive constant $M_{5}$, that

$$
I_{5} \leq M_{5}\left|t_{2}-t_{1}\right|^{2 \alpha}
$$

Therefore, we conclude that all $I_{i}, i=3,4,5$, converge to zero when $t_{2} \rightarrow t_{1}$. Thus, from Cauchy's convergence criterion, it yields $\lim _{t \rightarrow \beta^{-}} u(t)=u^{*}$. This finishes the proof of Lemma 4.2.

The second step of the proof of Theorem 4.1 consists in showing the following result.

Lemma 4.3 Function $u(t)$ is continuable.

Proof As $S$ is a closed subset, we can say that $\left(\beta, u^{*}\right) \in S$. Define $u(\beta)=u^{*}$. Hence, $u(t) \in$ $C[0, \beta]$. Then we define the operator $K$ by

$$
(K v)(t)=u_{1}+\frac{\lambda}{\Gamma(2 \alpha)} \int_{\beta}^{t}(t-s)^{2 \alpha-1} \frac{f(s, v(s))}{\left(\int_{0}^{t} f(x, v) d x\right)^{2}} d s,
$$


where

$$
u_{1}=u_{0}+\frac{\lambda}{\Gamma(2 \alpha)} \int_{0}^{\beta}(t-s)^{2 \alpha-1} \frac{f(s, v(s))}{\left(\int_{0}^{t} f(x, v) d x\right)^{2}} d s, \quad v \in C([\beta, \beta+1]), t \in[\beta, \beta+1] .
$$

Set

$$
E_{b}=\left\{(t, v): \beta \leq t \leq \beta+1,|v| \leq \max _{\beta \leq t \leq \beta+1}\left|u_{1}(t)\right|+b\right\}
$$

and

$$
E_{h}=\left\{v \in C[\beta, \beta+1]: \max _{t \in[\beta, \beta+h]}\left|v(t)-u_{1}(t)\right| \leq b, v(\beta)=u_{1}(\beta)\right\},
$$

where $h=\min \left\{\left(b\left(\frac{\lambda M}{\Gamma(2 \alpha+1) c_{1}^{2}}\right)^{-1}\right)^{\frac{1}{2 \alpha}}, 1\right\}$. Analogously to the proof of Theorem 3.2, we prove that $K$ is completely continuous on $E_{b}$. Indeed, let $\left\{v_{n}\right\} \subseteq C[\beta, \beta+h]$. Then $\| v_{n}-$ $v \|_{[\beta, \beta+h]} \rightarrow 0$ as $n \rightarrow+\infty$ and similar arguments to the one above for (7), allow us to declare that there exists a positive constant $c_{h}$ depending on $h$ such that

$$
\left\|K v_{n}-K v\right\|_{C[\beta, \beta+h]} \leq c_{h}\left\|v_{n}-v\right\|_{C[\beta, \beta+h]} .
$$

Hence, $\left\|\left(K v_{n}\right)(t)-(K v)(t)\right\|_{[\beta, \beta+h]} \rightarrow 0$ as $n \rightarrow+\infty$, which shows that the operator $K$ is continuous. We show that $K E_{h}$ is equicontinuous. For all $v \in E_{h}$, we have $(K v)(\beta)=u_{1}(\beta)$ and, in view of the choice of $h$, it follows from hypotheses $\left(H_{1}\right)$ and $\left(H_{2}\right)$ that

$$
\begin{aligned}
\left|(K v)(t)-u_{1}\right| & =\left|\frac{\lambda}{\Gamma(2 \alpha)} \int_{\beta}^{t}(t-s)^{2 \alpha-1} \frac{f(s, v(s))}{\left(\int_{0}^{t} f(x, v) d x\right)^{2}} d s\right| \\
& \leq \frac{\lambda M}{\Gamma(2 \alpha+1) c_{1}^{2}} h^{2 \alpha} \leq b .
\end{aligned}
$$

Therefore, we get $K E_{h} \subset E_{h}$. Furthermore, for any $v \in E_{h}$ and $\beta \leq t_{1} \leq t_{2} \leq \beta+h$, we have

$$
(K v)\left(t_{1}\right)-(K v)\left(t_{2}\right)=I_{6}+I_{7} .
$$

By a calculation analogous to the earlier one, there exists a positive constant $M_{6}$ such that

$$
\begin{aligned}
I_{6}= & \frac{\lambda}{\Gamma(2 \alpha)} \int_{0}^{\beta}\left(t_{2}-s\right)^{2 \alpha-1} \frac{f(s, v(s))}{\left(\int_{0}^{t_{2}} f(x, v) d x\right)^{2}} d s \\
& -\frac{\lambda}{\Gamma(2 \alpha)} \int_{0}^{\beta}\left(t_{1}-s\right)^{2 \alpha-1} \frac{f(s, v(s))}{\left(\int_{0}^{t_{1}} f(x, v) d x\right)^{2}} d s \\
\leq & \frac{\lambda}{\Gamma(2 \alpha)} \mid \int_{0}^{\beta}\left(\left(t_{1}-s\right)^{2 \alpha-1}-\left(t_{2}-s\right)^{2 \alpha-1}\right) \frac{f(s, v(s))}{\left(\int_{0}^{t_{1}} f(x, v) d x\right)^{2}} d s \\
& +\frac{\lambda}{\Gamma(2 \alpha)} \int_{0}^{\beta}\left(t_{2}-s\right)^{2 \alpha-1}\left(\frac{f(s, v(s))}{\left(\int_{0}^{t_{1}} f(x, v) d x\right)^{2}} d s-\frac{f(s, v(s))}{\left(\int_{0}^{t_{2}} f(x, v) d x\right)^{2}} d s\right) \mid \\
\leq & M_{6}\left\{\left|\left(t_{2}-\beta\right)^{2 \alpha}-\left(t_{1}-\beta\right)^{2 \alpha}+t_{1}^{2 \alpha}-t_{2}^{2 \alpha}\right|+\left|t_{2}-t_{1}\right|\right\} .
\end{aligned}
$$


An analogous treatment as in (8)-(10) yields the existence of a positive constant $M_{7}$ such that

$$
\begin{aligned}
I_{7}= & \frac{\lambda}{\Gamma(2 \alpha)} \int_{\beta}^{t_{2}}\left(t_{2}-s\right)^{2 \alpha-1} \frac{f(s, v(s))}{\left(\int_{0}^{t_{2}} f(x, v) d x\right)^{2}} d s \\
& -\frac{\lambda}{\Gamma(2 \alpha)} \int_{\beta}^{t_{1}}\left(t_{1}-s\right)^{2 \alpha-1} \frac{f(s, v(s))}{\left(\int_{0}^{t_{1}} f(x, v) d x\right)^{2}} d s \\
\leq & M_{7}\left\{\left|\left(t_{1}-\beta\right)^{2 \alpha}-\left(t_{2}-\beta\right)^{2 \alpha}\right|+\left|t_{2}-t_{1}\right|+2\left|t_{2}-t_{1}\right|^{2 \alpha}\right\} .
\end{aligned}
$$

Since the right side of inequalities (11) and (12) go to zero as $t_{2} \rightarrow t_{1}$, we deduce that $\left\{(K v)(t): v \in E_{h}\right\}$ is equicontinuous. Consequently, $K$ is completely continuous. Then Schauder's fixed point theorem can be applied to see that the operator $K$ has a fixed point $\tilde{u}(t) \in E_{h}$. On other words, we have

$$
\begin{aligned}
\tilde{u}(t) & =u_{1}+\frac{\lambda}{\Gamma(2 \alpha)} \int_{\beta}^{t}(t-s)^{2 \alpha-1} \frac{f(s, \tilde{u}(s))}{\left(\int_{0}^{t} f(x, \tilde{u}(x)) d x\right)^{2}} d s \\
& =u_{0}+\frac{\lambda}{\Gamma(2 \alpha)} \int_{0}^{t}(t-s)^{2 \alpha-1} \frac{f(s, \tilde{u}(s))}{\left(\int_{0}^{t} f(x, \tilde{u}(x)) d x\right)^{2}} d s, \quad t \in[\beta, \beta+h],
\end{aligned}
$$

where

$$
\tilde{u}(t)= \begin{cases}u(t), & t \in(0, \beta] \\ \tilde{u}(t), & t \in[\beta, \beta+h] .\end{cases}
$$

It follows that $\tilde{u}(t) \in C[0, \beta+h]$ and

$$
\tilde{u}(t)=u_{0}+\frac{\lambda}{\Gamma(2 \alpha)} \int_{0}^{t}(t-s)^{2 \alpha-1} \frac{f(s, \tilde{u}(s))}{\left(\int_{0}^{t} f(x, \tilde{u}(x)) d x\right)^{2}} d s .
$$

Therefore, according to Lemma 3.1, $\tilde{u}(t)$ is a solution of $(1)$ on $(0, \beta+h]$. This is absurd because $u(t)$ is noncontinuable. This completes the proof of Lemma 4.3 .

Theorem 4.1 follows from Lemmas 4.2 and 4.3.

Remark 4.1 Uniqueness of solution to problem (1) is easily derived from the proof of Theorem 4.1 for a well chosen $\lambda$.

\section{Global existence}

Now we provide two sets of sufficient conditions for the existence of a global solution for (1) (Theorems 5.2 and 5.3). We begin with an auxiliary lemma.

Lemma 5.1 Suppose that conditions $\left(H_{1}\right)-\left(H_{3}\right)$ hold. Let $u(t)$ be a solution of $(1)$ on $(0, \beta)$. If $u(t)$ is bounded on $[\tau, \beta)$ for some $\tau>0$, then $\beta=+\infty$.

Proof The result follows immediately from the results of Section 4 . 
Theorem 5.2 Suppose that conditions $\left(H_{1}\right)-\left(H_{3}\right)$ hold. Then (1) has a solution in $C([0,+\infty))$.

Proof The existence of a local solution $u(t)$ of (1) is ensured thanks to Theorem 3.2. We already know, by Lemma 3.1 , that $u(t)$ is a also a solution to the integral equation

$$
u(t)=u_{0}+\frac{\lambda}{\Gamma(2 \alpha)} \int_{0}^{t}(t-s)^{2 \alpha-1} \frac{f(s, u(s))}{\left(\int_{0}^{t} f(x, u(x)) d x\right)^{2}} d s .
$$

Suppose that the existing interval of the noncontinuable solution $u(t)$ is $(0, \beta), \beta<+\infty$. Then

$$
\begin{aligned}
|u(t)| & =\left|u_{0}+\frac{\lambda}{\Gamma(2 \alpha)} \int_{0}^{t}(t-s)^{2 \alpha-1} \frac{f(s, u(s))}{\left(\int_{0}^{t} f(x, u(x)) d x\right)^{2}} d s\right| \\
& \leq\left|u_{0}\right|+\frac{\lambda}{\Gamma(2 \alpha)} \frac{1}{\left(c_{1} t\right)^{2}} \int_{0}^{t}(t-s)^{2 \alpha-1}|f(s, u(s))| d s \\
& \leq\left|u_{0}\right|+\frac{\lambda}{\Gamma(2 \alpha)} \frac{1}{c_{1}^{2}} \int_{0}^{t} \frac{|u(s)|}{(t-s)^{1-2 \alpha}} d s .
\end{aligned}
$$

By Lemma 3.1, there exists a constant $k(\alpha)$ such that, for $t \in(0, \beta)$, we have

$$
|u(t)| \leq\left|u_{0}\right|+k\left|u_{0}\right| \frac{\lambda}{\Gamma(2 \alpha)} \frac{1}{c_{1}^{2}} \int_{0}^{t}(t-s)^{2 \alpha-1} d s,
$$

which is bounded on $(0, \beta)$. Thus, by Lemma 5.1, problem (1) has a solution $u(t)$ on $(0,+\infty)$.

Next we give another sufficient condition ensuring global existence for (1).

Theorem 5.3 Suppose that there exist positive constants $c_{3}, c_{4}$ and $c_{5}$ such that $c_{3} \leq$ $|f(s, x)| \leq c_{4}|x|+c_{5}$. Then (1) has a solution in $C([0,+\infty))$.

Proof Suppose that the maximum existing interval of $u(t)$ is $(0, \beta), \beta<+\infty$. We claim that $u(t)$ is bounded on $[\tau, \beta)$ for any $\tau \in(0, \beta)$. Indeed, we have

$$
\begin{aligned}
|u(t)| & =\left|u_{0}+\frac{\lambda}{\Gamma(2 \alpha)} \int_{0}^{t}(t-s)^{2 \alpha-1} \frac{f(s, u(s))}{\left(\int_{0}^{t} f(x, u(x)) d x\right)^{2}} d s\right| \\
& \leq\left|u_{0}\right|+\frac{\lambda}{\Gamma(2 \alpha)} \frac{1}{\left(c_{1} \tau\right)^{2}} \int_{0}^{t}(t-s)^{2 \alpha-1}|f(s, u(s))| d s \\
& \leq\left|u_{0}\right|+\frac{\lambda}{\Gamma(2 \alpha)} \frac{c_{3}}{\left(c_{1} \tau\right)^{2}} \int_{0}^{t}(t-s)^{2 \alpha-1} d s+\frac{\lambda}{\Gamma(2 \alpha)} \frac{c_{2}}{\left(c_{1} \tau\right)^{2}} \int_{0}^{t} \frac{|u(s)|}{(t-s)^{1-2 \alpha}} d s .
\end{aligned}
$$

If we take

$$
w(t)=\left|u_{0}\right|+\frac{\lambda}{\Gamma(2 \alpha)} \frac{c_{3}}{\left(c_{1} \tau\right)^{2}} \int_{0}^{t}(t-s)^{2 \alpha-1} d s,
$$

which is bounded, and

$$
a=\frac{\lambda c_{2}}{\Gamma(2 \alpha)} \frac{1}{\left(c_{1} \beta\right)^{2}}
$$


it follows, in accordance with Lemma 2.3, that $v(t)=|u(t)|$ is bounded. Thus, by Lemma 5.1, (1) has a solution $u(t)$ on $(0,+\infty)$.

\title{
6 Conclusion
}

In our paper we consider a prototype of electrical conductivity that depends strongly in both time and temperature. The model relates to modern developments of thermistors, where fractional PDEs have a crucial role. It turns out that available computational methods are not theoretically sound in the sense they rely on results of local existence. The main novelty of our paper is that we prove global existence for a nonlocal thermistor problem with fractional differentiation in the Caputo sense. Moreover, we extend some results of continuation and global existence to the fractional order initial value thermistor problem. The proofs rely on Schauder's fixed point theorem. We trust that our results will have a positive impact on the application of computer mathematics to fractional thermistor devices.

\author{
Acknowledgements \\ The authors were supported by the Center for Research and Development in Mathematics and Applications (CIDMA) of \\ University of Aveiro, through Fundação para a Ciência e a Tecnologia (FCT), within project UID/MAT/04106/2013. They are \\ grateful to two anonymous referees, for several comments and suggestions of improvement.

\section{Competing interests} \\ The authors declare that they have no competing interests. \\ Authors' contributions \\ All three authors contributed equally to this work. They all read and approved the final version of the manuscript.

\section{Author details} \\ 'Department of Mathematics, AMNEA Group, Faculty of Sciences and Techniques, Moulay Ismail University, B.P. 509, \\ Errachidia, Morocco. ${ }^{2}$ Department of Mathematics, R\&D Unit CIDMA, University of Aveiro, Aveiro, 3810-193, Portugal.
}

\section{Publisher's Note}

Springer Nature remains neutral with regard to jurisdictional claims in published maps and institutional affiliations.

Received: 21 July 2017 Accepted: 31 October 2017 Published online: 15 November 2017

\section{References}

1. Almeida, R, Pooseh, S, Torres, DFM: Computational Methods in the Fractional Calculus of Variations. Imperial College Press, London (2015)

2. Nishimoto, K: An Essence of Nishimoto's Fractional Calculus. Descartes Press, Koriyama (1991)

3. Aubin, J-P, Lygeros, J, Quincampoix, M, Sastry, S, Seube, N: Impulse differential inclusions: a viability approach to hybrid systems. IEEE Trans. Autom. Control 47(1), 2-20 (2002)

4. Kilbas, AA, Srivastava, HM, Trujillo, JJ: Theory and Applications of Fractional Differential Equations. North-Holland Mathematics Studies, vol. 204. Elsevier, Amsterdam (2006)

5. Miller, KS, Ross, B: An Introduction to the Fractional Calculus and Fractional Differential Equations. Wiley, New York (1993)

6. Podlubny, I: Fractional Differential Equations. Mathematics in Science and Engineering, vol. 198. Academic Press, San Diego (1999)

7. Samko, SG, Kilbas, AA, Marichev, Ol: Fractional Integrals and Derivatives. Gordon \& Breach, Yverdon (1993). Translated from the 1987 Russian original

8. Machado, JT, Kiryakova, V, Mainardi, F: Recent history of fractional calculus. Commun. Nonlinear Sci. Numer. Simul. 16(3), 1140-1153 (2011)

9. Baleanu, D, Golmankhaneh, AK, Golmankhaneh, AK, Nigmatullin, RR: Newtonian law with memory. Nonlinear Dyn. 60(1-2), 81-86 (2010)

10. Baleanu, D, Golmankhaneh, AK, Nigmatullin, R, Golmankhaneh, AK: Fractional Newtonian mechanics. Cent. Eur. J. Phys. 8(1), 120-125 (2010)

11. Baleanu, D, Diethelm, K, Scalas, E, Trujillo, JJ: Fractional Calculus. Series on Complexity, Nonlinearity and Chaos, vol. 5 World Scientific, Hackensack (2017)

12. Ortigueira, MD: Fractional Calculus for Scientists and Engineers. Lecture Notes in Electrical Engineering, vol. 84 Springer, Dordrecht (2011)

13. Kumar, D, Singh, J, Baleanu, D: A new analysis for fractional model of regularized long-wave equation arising in ion acoustic plasma waves. Math. Methods Appl. Sci. 40, 5642-5653 (2017) 
14. Srivastava, HM, Kumar, D, Singh, J: An efficient analytical technique for fractional model of vibration equation. Appl. Math. Model. 45, 192-204 (2017)

15. Singh, J, Kumar, D, Qurashi, MA, Baleanu, D: A new fractional model for giving up smoking dynamics. Adv. Differ. Equ. 2017, 88 (2017)

16. Singh, J, Kumar, D, Qurashi, MA, Baleanu, D: A novel numerical approach for a nonlinear fractional dynamical model of interpersonal and romantic relationships. Entropy 19(7), 375 (2017)

17. Sidi Ammi, MR, Torres, DFM: Numerical analysis of a nonlocal parabolic problem resulting from thermistor problem. Math. Comput. Simul. 77, 291-300 (2008)

18. Sidi Ammi, MR, Torres, DFM: Optimal control of nonlocal thermistor equations. Int. J. Control 85(11), 1789-1801 (2012)

19. Kwok, K: Complete Guide to Semiconductor Devices. McGraw-Hill, New york (1995)

20. Maclen, ED: Thermistors. Electrochemical publication, Glasgow (1979)

21. Hilfer, R: Applications of Fractional Calculus in Physics. World Scientific, River Edge (2000)

22. Lakshmikantham, V, Leela, S, Vasundhara Devi, J: Theory of Fractional Dynamic Systems. Cambridge Scientific Publishers, Cambridge (2009)

23. Arara, A, Benchohra, M, Hamidi, N, Nieto, JJ: Fractional order differential equations on an unbounded domain. Nonlinear Anal. 72(2), 580-586 (2010)

24. Li, C, Sarwar, S: Existence and continuation of solutions for Caputo type fractional differential equations. Electron. J. Differ. Equ. 2016, 207 (2016)

25. Sidi Ammi, MR, Torres, DFM: Existence and uniqueness of a positive solution to generalized nonlocal thermistor problems with fractional-order derivatives. Differ. Equ. Appl. 4(2), 267-276 (2012)

26. Sidi Ammi, MR, Torres, DFM: Galerkin spectral method for the fractional nonlocal thermistor problem. Comput. Math. Appl. 73(6), 1077-1086 (2017)

27. Sidi Ammi, MR, Torres, DFM: Existence and uniqueness results for a fractional Riemann-Liouville nonlocal thermistor problem on arbitrary time scales. J. King Saud Univ. Sci. (2017) in press. doi:10.1016/j.jksus.2017.03.004

28. Li, Y, Chen, Y, Podlubny, I: Mittag-Leffler stability of fractional order nonlinear dynamic systems. Automatica 45(8), 1965-1969 (2009)

29. Li, C, Deng, W: Remarks on fractional derivatives. Appl. Math. Comput. 187(2), 777-784 (2007)

30. Li, CP, Zeng, FH: Numerical Methods for Fractional Differential Calculus. Chapman \& Hall, Boca Raton (2015)

31. Caputo, M: Linear models of dissipation whose $Q$ is almost frequency independent. II. Geophys. J. R. Astron. Soc 13(5), 529-539 (1967)

32. Caputo, M: Elasticità e Dissipazione. Zanichelli, Bologna (1969)

33. Caputo, M, Mainardi, F: Linear models of dissipation in anelastic solids. Riv. Nuovo Cimento (Ser. II) 1, 161-198 (1971)

34. Mainardi, F: Fractional calculus: some basic problems in continuum and statistical mechanics. In: Fractals and Fractional Calculus in Continuum Mechanics (Udine, 1996), CISM Courses and Lect., vol. 378. Springer, Vienna (1997)

35. Henderson, J, Ouahab, A: Impulsive differential inclusions with fractional order. Comput. Math. Appl. 59(3), 1191-1226 (2010)

36. Ye, $\mathrm{H}, \mathrm{Gao}, \mathrm{J}$, Ding, Y: A generalized Gronwall inequality and its application to a fractional differential equation. J. Math. Anal. Appl. 328(2), 1075-1081 (2007)

37. Kou, C, Zhou, H, Li, C: Existence and continuation theorems of Riemann-Liouville type fractional differential equations. Int. J. Bifurc. Chaos Appl. Sci. Eng. 22(4), 1250077 (2012)

\section{Submit your manuscript to a SpringerOpen ${ }^{\circ}$ journal and benefit from:}

- Convenient online submission

- Rigorous peer review

- Open access: articles freely available online

- High visibility within the field

- Retaining the copyright to your article

Submit your next manuscript at $\boldsymbol{~ s p r i n g e r o p e n . c o m ~}$ 